\title{
From many deaths to some few cases of drug-resistant tuberculosis: travelling with the systems quality improvement model in Lacs Health District, Togo
}

\author{
Kossivi Agbelenko Afanvi \\ Lacs Health District and UNIVERSITE DE LOME (Togo)
}

\begin{abstract}
The ultimate goal of every tuberculosis (TB) treatment program is a high treatment success rate. Treatment success is extremely important because, when the rate is high, it significantly contributes to declining numbers of new cases by reducing the number and period of infectious cases, TB morbidity and mortality, and prevents the emergence of resistant strains. Our aim was to decrease TB mortality by increasing pulmonary TB patients' treatment success rate to at least $85 \%$ in Lacs Health District by end of July 2014.
\end{abstract}

A systems and dialogic analysis of the public health system related to TB patients' treatment revealed that it was not performing well; we found weak coverage and quality of TB services, a poorly-functioning TB health information system, poor-performing health workforce, poor availability of HIV tests and antiretroviral for TB patients, and low degree of patients' participation in their care. We redesigned the system to correct those weaknesses. The effectiveness of these changes was monitored using plan, do, study, act (PDSA) cycles. We increased TB patient success rate from $80 \%$ to $95 \%$ between February 2012 and July 2014 . The mortality rate dropped from $13 \%$ to $3 \%$ and the failure to follow-up rate dropped from $3 \%$ to $2 \%$.

In conclusion, district health systems performance depends on factors such as the closeness of services to population; skilled workforce; the ability to collect and analyze data and use information for action; population empowerment, and good management and improvement capabilities of management team especially the public health director. High TB patients' success rate depends also on the availability of antiretroviral drugs. It is highly important that every district health management team member develops improvement capabilities.

\section{Problem}

Tuberculosis is the second leading cause of death among communicable diseases worldwide, killing nearly two million people each year, with most cases in less-developed countries.[1] Even though there have been improvements in tuberculosis control over the past two decades, its incidence has increased in Africa, mainly as a result of the burden of HIV infection.[2,3] Without treatment, TB mortality rates are high.

In 1994, the internationally recommended control strategy (later named DOTS strategy[4]) was launched as part of this Stop TB Strategy. It is expected to (a) detect, by 2005 , at least $70 \%$ of new sputum smear-positive TB cases and cure at least $85 \%$ of these cases and (b) reduce TB prevalence and death rates by $50 \%$ relative to 1990 by 2015. In Lacs Health District in Togo, TB treatment success rate was $80 \%$ in 2011 and the lethality rate was $13 \%$. As an autograph in the book 'Management of tuberculosis: a guide to the essentials of good practice - 6th edition' (given to the corresponding author at the closing ceremonies of the 19th International Course on Tuberculosis Management in Cotonou in September 2011), Dr. Arnaud Trébucq and Prof. Martin Gninafon wrote, "To Kossivi, whom we expect to quickly apply in Lacs Health District all the best directives discussed during the 19th International Course on Tuberculosis Management in Cotonou. All the best." When he handed him the book, Dr. Arnaud Trébucq said,
"The starting point is to reduce TB death."

Our aim in this quality improvement project was to decrease TB mortality by increasing pulmonary TB patients' treatment success rate to at least 85\% in Lacs Health District by the end of July 2014.

\section{Background}

According to World Health Organization (WHO), the most costeffective way to stop the spread of TB in communities with a high incidence is by curing it using the DOTS strategy.[4] Key components of the DOTS strategy include: (i) government commitment (including political will at all levels, and establishment of a centralized and prioritized system of TB monitoring, recording and training); (ii) case detection by sputum smear microscopy; (iii) standardized free treatment regimen of six to eight months directly observed by a healthcare worker or community health worker for at least the first two months; (iv) a regular, uninterrupted drug supply; (v) a standardized recording and reporting system that allows assessment of treatment results. To achieve this Stop TB Strategy, six implementation approaches were proposed[4]: (i) pursue highquality DOTS expansion and enhancement; (ii) address TB/HIV, multi-drug resistant TB strains (MDR-TB) and other challenges; (iii) contribute to health system strengthening; (iv) engage all care providers; (v) empower people with TB, and communities; and (vi) enable and promote research. 
BMJ Quality Improvement Reports

The goals of TB treatment are to ensure cure without relapse, prevent death, stop transmission, and to prevent the emergence of drug resistance.[1] According to Cochrane reviews in 2003 [5] and $2007,[6]$ the effects of direct observation on cure or treatment completion were similar to those of self-administered treatment. There is also no difference whether the treatment was directly observed by a family member or community health worker or healthcare provider at clinic.

In Togo, the recommended treatment regimens have two phases [7]: (i) an initial intensive phase of two months designed to kill actively growing and semi-dormant bacilli and (ii) a continuation phase of four months. Togo follows WHO-recommended treatment regimens as described by Frieden.[1] For the doses, route of administration, and mode of action of the drugs, one can read Frieden (2003) and PNLT (2008). At the beginning of the treatment, the support of the patient to the treatment must be obtained. One must emphasize the nature of the disease, the contamination mode, the duration of the treatment, the link between TB and HIV, the bacteriological exams, and the necessity treatment observation either by a health worker or a designated supervisor.[7]

\section{Baseline measurement}

One of the key elements in improvement is to control variation. Before deciding on which change to test, we must know if the system was stable or in a state of statistical control or unstable with special causes of variation.[8] To do so, we plotted on a Shewhart $P$ Chart data related to TB patient treatment success rate from January 2010 to January 2012 using QI-Charts v. 1.0.39 from Scoville Associates. Before the project, the TB care system in the district was stable because it exhibits only common cause variation. The chart presented on figure 1 revealed that: (i) there was no single point outside the control limits; (ii) there was no run of eight or more points in a row above (or below) the centerline; (iii) there were no six consecutive points increasing (trend up) or decreasing (trend down) (even though this third rule should be used with cautious in our case because of varying limits); (iv) we did not have two out of three consecutive points near (outer one-third) a control limit. The point of January 2011 is not in the outer one-third but in the limit inner two-third and outer one-third. The one of March 2011 is inside the inner two-third. This means that we were seeing only variation inherent in the current TB patient care process.

To understand how the TB care system was functioning, we created a systems analysis using WHO health systems building blocks [9] and the structured thinking and communication developed by Management Consulting Institute.[10] The results of the analysis (table 1) revealed that all the six subsystems were not wellfunctioning: service delivery sub-system was the least wellfunctioning, followed by human resources for health $(\mathrm{HRH})$, health information system, medical products, vaccines, and technologies and health financing. Even if leadership and governance was the least affected, the dysfunction related to low degree of patient and family participation to their care process. The cause and effect analysis of the high mortality rate observed is presented in figure 2 .

See supplementary file: ds5685.docx - "From many deaths to some few cases of drug-resistant tuberculosis_Attachments"

\section{Design}

In a system that exhibits only the common cause of variation, the improvement strategy must involve [8]: (i) process redesign or identification of aspects of the process to change to make it perform better; (ii) testing those changes using the PDSA cycle; and (iii) implementing successful changes using the PDSA cycle.

We redesigned the TB care system by increasing treatment services availability and quality, and information analysis and use. We used a model we called systems quality improvement model (SQIM) (figure 3). We merged the IHI Breakthrough series model,[11] the Dialogic Change Model [12] and Deming's Leadership principles.[13] In this model, the system was analyzed to determine whether it was stable or not. If unstable, special causes of variation were analyzed and corrective actions tested and implemented on an ongoing basis using PDSA cycles until the system becomes stable. In the state of statistical control, the poorly performing parts of the system were analyzed and redesigned; changes were tested and implemented using PDSA cycles until the system was sustainable as a whole.

From the beginning and at each step and stage, dialogue was embedded in the process as the motor oil for progress. At the beginning, the stakeholder dialogues were geared towards consultation since we needed the viewpoints, experiences, and expertise of all stakeholders. During the test and implementation, stakeholders were active in achieving planned results. The job of the leader was to transform the system. He possessed knowledge and was transformed by the Institute for Healthcare Improvement through its Open School courses. To accomplish this transformation, the leader had a theory: he knew that transformation would enhance TB program performance with obvious outcomes at individual patient level, family and other stakeholders' level, and the organizational level. The leader was compelled to accomplish the transformation because he was the district director and he had a direct invitation from Dr. Arnaud Trébucq and Prof. Martin Gninafon at the closing ceremonies of the 19th International Course on Tuberculosis Management in Cotonou. Finally, as a physician, he was a practical man. He knew step by step how to alleviate TB disease burden on the population.

All health centres and dispensaries with at least a registered nurse were transformed to a TB diagnosis and treatment services provider. In addition, we modeled the directly observed treatment short-course to an empowered directly observed treatment shortcourse. Each newly diagnosed TB patient was screened for HIV and was asked to identify a family member who can supervise the treatment. The degree of patients and family participation in the care process was increased by empowering them to become agents of their own care: they chose themselves, according to their location and will, the health centre in which they wanted to receive their treatment. They were also empowered to recognize TB drugs side effects; what to do when they were in face of those side effects and when to seek for care. The treatment was directly observed either by a healthcare provider, a community health worker or a 
selected family member.

During the two month intensive phase, patients who were at a distance less than $2.5 \mathrm{~km}$ of a healthcare facility go there every morning to take their medication. But those at a distance of or more than $2.5 \mathrm{~km}$ were provided drugs for one week and should go back to the visit health centers two days before the end of their drugs for renewal. Bacteriological examination of sputum was done at the end of the second month and patient entered the continuation phase. In the continuation phase which lasts four months, all patients received a provision of one month and half ( 45 days). Patients visited health facilities two days before the end of their medicine for renewal. At the end of the fifth month, the second bacteriological sputum control was performed. If the result was negative, patients received another provision of one month. But if positive, the patient was considered in therapeutic failure and his treatment involved other drugs. We also increased the availability of HIV screening reagent and antiretroviral drugs.

\section{Strategy}

System quality improvement model (SQIM) uses a transdisciplinary team work approach. To select the team (called container in dialogic model), it is important to perform stakeholders analysis.[12] Table 2 and figure 4 present results of the analysis. We integrated to the container stakeholders that had high to medium interest and high to medium impact. The team who ran the test comprised healthcare providers, researchers, managers, patients, and one of their family member and the civil society. Table 3 displays their role and responsibilities in the project. Because monthly TB smear positive cases in the district vary from three to 15 and from different health centres, our project took place in all health centers with at least one trained registered nurse.

PDSA cycle 1: The test took place on an ongoing basis from February 2012. According to the type of Shewhart control chart and the initial value of non-conforming, some basic rules must be satisfied.[8] The baseline treatment success rate was $80 \%$, implying $20 \%$ non-conforming. So we needed at least seven months to get a $\mathrm{P}$ chart that could be useful; at least 15 months to follow the common guideline for $\mathrm{P}$ charts; and at least 36 months to get a $\mathrm{P}$ chart with a lower control limit. We predicted to achieve every month at least $85 \%$ of TB patients treatment success rate; $5 \%$ or less mortality rate among TB smear positive patient; and $4 \%$ or less failure to follow-up rate among TB patients. Even though the average treatment success rate increased a lot and the average mortality and failure to follow-up rates decreased, we noticed a anomalous increase in the mortality rate due to TB-HIV co-infection.

PDSA cycle 2: In our effort to learn from this anomaly, we found that despite the recommendations to give priority to TB-HIV coinfected patients and systematically put them under ART, this was not done every time. During a supervision of the central level, the report was discussed and a formal order was given to the ART providing centre to give priority to TB-HIV co-infected patients. We decided to test whether systematically putting TB-HIV co-infected patients under antiretroviral treatment could decrease to $3 \%$ or less TB mortality and increase to at least $95 \%$ TB treatment success rate every month. Each healthcare provider tested every newly diagnosed TB patient for HIV. All TB-HIV co-infected patients were transferred to the TB focal point who had the entire responsibility to provide him with ART and monitor the treatment. When the coinfected patient successfully ended his TB treatment, he was transferred to an HIV Care Centre. The result of the test was successful but we faced another special cause of variation due to TB drug resistance.

PDSA cycle 3: Drug resistance is a huge issue efforts to stop TB are facing all over the world. There are several types of drug resistance [7,14]: monoresistance (resistance of mycobacterium tuberculosis to one TB drug), polyresistance (resistance of $\mathrm{M}$ tuberculosis to at least two drugs without simultaneous resistance to isoniazid and rifampicin), multi-drug resistance (simultaneous resistance of $\mathrm{M}$ tuberculosis to isoniazid and rifampicin) and Extensively drug-resistant (resistance of $\mathrm{M}$ tuberculosis to at least isoniazid and rifampicin, any fluoroquinolone, and at least one of three injectable second-line drugs (amikacin, capreomycin, or kanamycin)).

Drug resistance is preventable and avoidable if there is good compliance and good patient monitoring. The problem is whether it is possible to depict early signs so that appropriate action is taken the answer is "Yes"! In developed countries this is corrected since through polymerase chain reaction (PCR) and bacteriological culture, resistant strains are identified and effective drugs prescribed at the beginning of the treatment.

Unfortunately, in Togo PCR and bacteriological culture are performed only when there is treatment failure due to the lack of resources. The alternative (albeit less specific) method is to rely on clinical signs. Normally, two weeks after a well-observed treatment, there is a significant decrease in clinical signs. Based on such knowledge, treatment failure can be suspected if the patient still presents clinical symptoms like cough after the initial phase of two months. So, in presence of an evocative cough after two months of well-observed treatment, one can ask for PCR and bacteriological culture. We tested our changes to see whether it could help improve and sustain our TB program performance.

\section{Results}

The outcome measure of our project was TB treatment success rate. It was monitored every month to assess progress and success. In addition to monitoring the treatment success rate, we also assessed mortality rate as process measure and treatment failure rate as balancing measures. Changes were an improvement if: (i) there was a single point outside the control limits; (ii) there was a run of eight or more points in a row above (or below) the centerline; (iii) there were six consecutive points increasing (trend up) or decreasing (trend down) (even though this third rule should be used with cautious in our case because of varying limits); (iv) we had two out of three consecutive points near (outer one-third) a control limit. Figures 5 to 7 displayed the results.

After the first PDSA cycle, the average treatment success rate moved from 80\% to 90\% between February and September 2012. 
From February to May 2012 we noticed a special cause signaling an improvement: two out of three consecutive points were on the upper control limit. In September 2012, we noticed a decrease in the success rate to $67 \%$. Even though it was not outside nor near the lower control limit, we thought it was important to better understand what happened. After analysis, we realized it was due to an increase in mortality rate from TB-HIV co-infection. The average mortality rate dropped from $13 \%$ to $5 \%$. From february to May 2012, we noticed no deaths: four consecutive points were on the lower control limit indicating an improvement. From July to September 2012, there was another special cause: two out of three consecutive points were near (outer one-third) the control limits indicating an abnormal increase in the TB mortality rate due to TBHIV co-infection. Initially, failure to follow-up rate among TB patients was below $4 \%$, the prediction level. it dropped from $3 \%$ to $2 \%$ and from April 2012, no failure to follow-up was noticed among TB patients.

After the second PDSA cycle, the average treatment success rate increased from $90 \%$ to $94 \%$. Between October 2012 and February 2013, there were four out of five consecutive points on the upper control limit indicating an improvement. In March 2013, another special cause (drug resistance) decreased the success rate to $67 \%$. From January 2013, we noticed no deaths. The treatment failure to follow-up rate improved dramatically. The performance achieved since April 2012 was maintained.

The third PDSA cycle helped improve our TB program performance: we achieved on average $95 \%$ treatment success rate. The success was not sustainable because we noticed an increase in TB death on October and December 2013: on October, the only one case that died was not diagnosed earlier and the December case came from Nigeria. Since then, death is no more a problem; the challenge relied on drug resistance.

See supplementary file: ds5700.docx - "Results of the project"

\section{Lessons and limitations}

In our first PDSA we were looking for which combinations of changes could help achieve at least $85 \%$ of treatment success rate. We predicted to achieve at least $85 \%$ treatment success rate; $5 \%$ or less mortality rate and $4 \%$ or less failure to follow-up rate among TB patients every month. We dealt with TB-HIV co-infection and drug resistance in PDSA cycles 2 and 3.

Globally, we surpassed our predictions but we realized that the system could not sustain without systematically putting TB-HIV coinfected under antiretroviral and well-managing drug resistance.

The big challenge of this project was early diagnosis of TB drug resistance. Based on this knowledge, the National TB Conrol Program, PNLT, included in its strategic plan for 2015 to 2019, systematic PCR to each newly diagnosed tuberculosis case before treatment.

We surpassed the $85 \%$ treatment success rate recommended by the WHO.[4] Successful treatment of individual cases reduces TB morbidity and mortality, reduces the number and period of infectious cases, and prevent the emergence of resistant strains. This target was based on epidemiological modeling and suggests that achievement of an $85 \%$ cure rate and $70 \%$ case detection will reduce the prevalence of infectious (sputum smear-positive) TB cases, the number of infected contacts, and the incidence of infectious cases. Further to this, the achievement of these targets for case detection and cure is expected to reduce the annual TB incidence rate by 7 to $12 \%$ per year, in the absence of HIV coinfection.[4]

DOTS, as recommended by the WHO, is a practical and powerful strategy to overcome the burden of tuberculosis in resource-limited area. The observation can be done either by a healthcare worker, a family member, or community health worker without effect on its effectiveness as showed by Cochrane Reviews in 2003 [5] and 2007.[6] By including family members, we increased their empowerment to positively live with TB patients, offer them assistance, better understand the disease and its treatment; this will help to reduce the stigmatization TB patients face in their daily lives.

The dialogic approach we used to empower patients and their family members was crucial for the achievement. The most important common features of stakeholder dialogues are structured conversations about the issues TB patient were facing. These conversations improve planning and decision-making; solve problems; contribute to finding innovative solutions, and to designing and implementing joint intervention for change.

Stakeholder dialogues are vital stepping stone in achieving a common goal, and lead to a practical outcome that could not have been achieved otherwise and that can more easily be implemented because all stakeholders involved experience a higher degree of ownership.[12]

The second challenge of TB care is HIV co-infection. Lots have been written about the impact of the co-infection on tuberculosis and HIV diseases epidemiology and their control programs. [2, 3, 4, $15,16]$ Among important actions is antiretroviral treatment and failure to successfully integrate HIV and tuberculosis control will threaten the viability of both programmes.

The third and most difficult to overcome is drug resistance. From the advent of tuberculosis chemotherapy in the 1940s, hints of resistance were evident. Even though technology allows us to early diagnose and cure TB drug resistance, the limited laboratory capacities and staff training in resource-limited settings currently preclude their widespread use.[14] Preventing transmission of $M$ tuberculosis relies on an accurate and rapid diagnosis. A key barrier to tuberculosis control is that current case detection rates are low.

While waiting to find a solution to the availability of technologies to early diagnose drug resistance, we can try to increase both case detection and cure rate. System quality improvement model (SQIM) offers an approach to achieve $95 \%$ treatment success rate without limited resource. SQIM is a merge of three scientifically proven models: IHI Breakthrough series model,[11] the Dialogic Change Model [12] and Deming's Leadership principles.[13] $\mathrm{IHI}$ Breakthrough series model includes the model of improvement,[17] 
another scientifically proven model to achieve high performance.

\section{Conclusion}

Health systems performance at district level depends on factors such as the closeness of services to population; skilled workforce; ability to collect and analyze data and use information for action; population empowerment and good management and improvement capabilities of management team especially the public health director. High TB patients' success rate depends also on the availability of antiretroviral drugs. We strongly suggest increasing improvement knowledge and capabilities in every district health management team member.

The success of the project was based on the systems analysis of the existing health system and the management and improvement capabilities of management team. Improvement capabilities helped to understand variation and team management or (better) stakeholders' management strengthens achievements from each step.

Undoubtedly medical management requires strong clinical management competencies (particularly clinical status monitoring) during care to depict early signs of treatment failure to be managed. Population empowerment should be the future focus of health system to achieve high and sustainable performance. Health systems should be managed as a system, taking into account all the six building blocks.

By raising TB patients' treatment success rate, we allow hope to eliminating TB in Lacs Health District. Other districts could use system quality improvement model to improve their health programs performance.

\section{References}

1. Frieden TR, Sterling TR, Munsiff SS, Watt $\mathrm{CJ}$ and Dye $\mathrm{C}$. Tuberculosis. Lancet 2003; 362:887-99.

2. Atun R, Raviglione Mo, Marais B, Zumla A. Tuberculosis control is crucial to achieve the MDGs. Lancet 2010; 376:940-41.

3. WHO. Global tuberculosis report 2013. Geneva: World Health Organization; 2013.

4. WHO. The Stop TB Strategy: building on and enhancing DOTS to meet the TB-related Millennium Development Goals. Geneva: World Health Organization; 2006.

5. Volmink J, Garner P. Directly observed therapy for treating tuberculosis. Cochrane Database Syst Rev; 20 January 2003. Issue 1. Art. No.: CD003343. DOI: 10.1002/14651858.CD003343.

6. Volmink J, Garner P. Directly observed therapy for treating tuberculosis. Cochrane Database of Systematic Reviews; 13 August 2007. Issue 4. Art. No.: CD003343. DOI: 10.1002/14651858.CD003343.pub3.

7. PNLT. Guide Technique du Programme National de Lutte contre la Tuberculose - 2ème Edition. Lomé : Programme National de Lutte contre la Tuberculose; 2008.
8. Provost LP, Murray SK. The health care data guide: learning from data for improvement. San Francisco: JosseyBass; 2011.

9. WHO. Everybody's Business. Strengthening health systems to improve health outcome. Geneva: World Health Organization; 2007.

10. MCl. Management consulting body of knowledge (MCBOK). Consulting Methods, 1st Edition. Cambridge: Management Consulting Institute; 2013.

11. IHI. The Breakthrough Series: IHI's Collaborative Model for Achieving Breakthrough Improvement. IHI Innovation Series white paper. Boston: Institute for Healthcare Improvement; 2003.

12. Kuenkel P, Gerlach S, Frieg V. Working with Stakeholder Dialogues - Key Concepts and Competencies for Achieving Common Goals. Norderstedt-Germany: Collective Leadership Institute; 2011.

13. Deming, WE. A system must be managed. [In] JN Orsini. The Essential Deming. Leadership Principles from the Father of Quality. San Francisco: McGraw-Hill; 2013.

14. Jassal M, Bishai WR. Extensively drug-resistant tuberculosis. Lancet Infect Dis 2009; 9:19-30.

15. Corbett EL, Marston B, Churchyard GJ, De Cock KM. Tuberculosis in sub-Saharan Africa: opportunities, challenges, and change in the era of antiretroviral treatment. Lancet 2006; 367:926-37.

16. Sileshi B, Deyessa N, Girma B, Melese M, Suarez P. Predictors of mortality among TB-HIV Co-infected patients being treated for tuberculosis in Northwest Ethiopia: a retrospective cohort study. BMC Infect Dis 2013; 13:297.

17. Langley GJ, Moen RD, Nolan KM, Nolan TW, Norman CL, Provost LP. The improvement guide: a practical approach to enhancing organizational performance - 2nd edition. San Francisco: Jossey-Bass; 2009.

\section{Declaration of interests}

Nothing to declare.

\section{Acknowledgements}

We would like to thank Essilivi Comlanvi Tsomo, RN, TB Focal Point in Lacs Health District.

\section{Ethical approval}

The study is an action-research aimed to improve Lacs Health District TB Program performance. It does not involve study on human subjects. All that is done was part of routine activities; the researchers just improved the way those activities were performed. 